\title{
An oscillation theorem for the nonlinear degenerate elliptic equation in the Heisenberg group
}

\section{Duan $\mathrm{Wu}^{1}$ and Pengcheng $\mathrm{Niu}^{1 *}$ (B)}

\section{"Correspondence:}

pengchengniu@nwpu.edu.cn ${ }^{1}$ School of Mathematics and Statistics, Northwestern Polytechnical University, Xi'an, Shaanxi 710129, P.R. China

\begin{abstract}
The aim of this paper is to study the oscillation of solutions of the nonlinear degenerate elliptic equation in the Heisenberg group $H^{n}$. We first derive a critical inequality in $H^{n}$. Based on it, we establish a Picone-type differential inequality and a Sturm-type comparison principle. Then we obtain an oscillation theorem. Our result generalizes the related conclusions for the nonlinear elliptic equations in $R^{n}$.

MSC: 35B05; 35J70; 35R03

Keywords: Heisenberg group; Nonlinear degenerate elliptic equation; Picone-type inequality; Sturm-type comparison principle; Oscillation
\end{abstract}

\section{Introduction}

In this paper, we give an oscillation theorem for the nonlinear degenerate elliptic equation in the Heisenberg group $H^{n}$

$$
\nabla_{H} \cdot\left(A(z, t) \nabla_{H} v(z, t)\right)+B^{T}(z, t) \nabla_{H} v(z, t)+C(z, t) f(v(z, t))=0
$$

and the corresponding homogeneous ordinary differential equation

$$
\left(p(r) u^{\prime}(r)\right)^{\prime}+b(r) u^{\prime}(r)+c(r) u(r)=0
$$

where $(z, t) \in \Omega, \Omega$ is the outer region in $H^{n}, \nabla_{H}$ denotes the Heisenberg gradient (see (2.1)), $r=|(z, t)|_{H},|(z, t)|_{H}$ denotes the norm in $H^{n}$ (see (2.4)), and $A(z, t), B(z, t), C(z, t)$, $f(v), p(r), b(r)$, and $c(r)$ are to be specified later.

The oscillation result obtained in this paper implies that if Eq. (1.2) is oscillatory. Then the judgement of oscillation for Eq. (1.1) can be transformed into comparing the relationship between the corresponding coefficients in Eqs. (1.1) and (1.2).

The oscillation properties for ordinary differential equations in the real line $R$ have been investigated by many authors with different methods (see $[1,5,6,11,12,16]$ and references therein). A well-known result was shown by Picone [11], in which an identity (now it is

(c) The Author(s) 2021. This article is licensed under a Creative Commons Attribution 4.0 International License, which permits use, sharing, adaptation, distribution and reproduction in any medium or format, as long as you give appropriate credit to the original author(s) and the source, provide a link to the Creative Commons licence, and indicate if changes were made. The images or other third party material in this article are included in the article's Creative Commons licence, unless indicated otherwise in a credit line to the material. If material is not included in the article's Creative Commons licence and your intended use is not permitted by statutory regulation or exceeds the permitted use, you will need to obtain permission directly from the copyright holder. To view a copy of this licence, visit http://creativecommons.org/licenses/by/4.0/. 
called the Picone identity) between

$$
\left(p(t) y^{\prime}(t)\right)^{\prime}+q(t) y(t)=0
$$

and

$$
\left(P(t) x^{\prime}(t)\right)^{\prime}+Q(t) x(t)=0
$$

was established, where $p(t), P(t) \in C^{1}([a, \infty), R), q(t), Q(t) \in C([a, \infty), R)$, and $a$ is a positive constant. Then the Sturm comparison principle for Eqs. (1.3) and (1.4) was derived. Finally, the oscillation theorem for Eq. (1.4) followed.

The oscillation criteria for linear or nonlinear partial differential equations have also been concerned extensively. Noussair [10] concluded some oscillation criteria for semilinear elliptic inequalities by the Riccati transformation. $\mathrm{Xu}$ [14] formulated oscillation theorems for elliptic equations with damping by using the way similar to [10]. For more results, we refer the readers to $[8,9,13]$ and references therein. Recently, Zhuang [15] obtained oscillation criteria for second-order nonlinear elliptic differential equations.

Motivated by the ideas in [10,11], and [15], in this paper, we deduce an oscillation criterion for (1.1) in $H^{n}$. The difficulty is that there is no good divergence formula in $H^{n}$ as in $R^{n}$. Fortunately, we find a good estimate (see Lemma 2.3) to help us build the criterion.

Before stating our main result, we introduce some notations and notions. For positive constants $a, a_{1}, a_{2}$, we denote

$$
\begin{aligned}
& G\left[a_{1}, a_{2}\right]=\left\{(z, t) \in H^{n}: a_{1} \leq|(z, t)|_{H} \leq a_{2}\right\}, \\
& G[a,+\infty)=\left\{(z, t) \in H^{n}:|(z, t)|_{H} \geq a\right\} .
\end{aligned}
$$

We say that $\Omega$ is an outer region in $H^{n}$ if there exists a positive constant $a_{0}$ such that $G\left[a_{0},+\infty\right) \subset \Omega$. Let us restrict our attention to the nontrivial solutions $v(z, t)$ of Eq. (1.1), that is, to the solutions $v(z, t)$ of (1.1) satisfying

$$
\sup \{|v(z, t)|:(z, t) \in \Omega\}>0 \text {. }
$$

A nontrivial solution to Eq. (1.1) is called oscillatory if it has arbitrarily large zeros; otherwise, it is called nonoscillatory. Equation (1.1) is called oscillatory if all its solutions are oscillatory.

Hereafter, we always assume that the following conditions are satisfied:

$\left(C_{1}\right)$ the coefficient matrix $A(z, t)=\left(a_{i j}(z, t)\right)_{2 n \times 2 n}$ is real symmetric positive definite with $a_{i j} \in C_{\text {loc }}^{2}(\Omega, R)$, the largest (necessarily positive) eigenvalue of $A(z, t)$ is denoted by $\lambda_{\max }(z, t)$, and there exists a function $\lambda(r) \in C^{1}\left(R^{+}, R^{+}\right)$such that

$$
\lambda(r) \geq \max _{|(z, t)|_{H}=r} \lambda_{\max }(z, t), \quad r>a_{0}
$$

$\left(C_{2}\right) B^{T}(z, t)=\left(b_{i}(z, t)\right)_{1 \times 2 n}, b_{i}(z, t) \in C_{\mathrm{loc}}^{2}(\Omega, R)$;

$\left(C_{3}\right) C(z, t) \in C_{\text {loc }}^{1}(\Omega, R)$;

$\left(C_{4}\right) f \in C^{1}(R, R), v f(v)>0$ and $f^{\prime}(v) \geq k>0$ for $v \neq 0$ and a constant $k$;

$\left(C_{5}\right) p(r) \in C^{1}\left(\left[a_{0}, \infty\right),(0, \infty)\right), b(r), c(r) \in C\left(\left[a_{0}, \infty\right), R\right)$. 
We denote

$$
Q(r)=\int_{S_{H}(o, r)}\left(C(z, t)-\frac{1}{4 k}\left(B^{T} A^{-1} B\right)(z, t)-\frac{1}{2 k} \nabla_{H} \cdot B(z, t)\right) d H,
$$

where $S_{H}(o, r)$ denotes the sphere in $H^{n}$ with center $o=(0,0)$ and radius $r$, and $d H$ denotes the $2 n$-dimensional Hausdorff measure in $R^{2 n+1}$ (see [3]);

$$
g(r)=\frac{\wp \alpha_{\wp} \lambda(r)}{k r^{-2 n-1}}
$$

where $\wp=2 n+2$ is the homogeneous dimension of $H^{n}$, and $\wp \alpha_{\wp}\left(\alpha_{\wp}\right.$ is a constant) is the area of unit sphere $S_{H}(o, 1)$ in $H^{n}$.

Now we state our main result.

Theorem 1.1 Assume that there exists $a>0$ such that for any $r \in[a,+\infty)$,

$$
\begin{aligned}
& p(r)>\left(4 r^{2}+1\right) g(r), \\
& Q(r) \geq \frac{b^{2}(r)}{4\left(p(r)-\left(4 r^{2}+1\right) c(r)\right)}+c(r),
\end{aligned}
$$

and the equality is not true everywhere in any subinterval of $[a,+\infty)$. Then Eq. (1.1) is oscillatory when Eq. (1.2) is oscillatory.

The paper is organized as follows. In Sect. 2, we collect some well-known results for the Heisenberg group and prove a good estimate. Section 3 is devoted to proofs of the Picone-type differential inequality and the Sturm-type comparison principle. The proof of Theorem 1.1 is presented in Sect. 4.

\section{Preliminaries}

The Heisenberg group $H^{n}$ is $R^{2 n+1}$ (or $C^{n} \times R$ ) endowed with the group law o defined by

$$
\bar{\xi} \circ \xi=\left(x+\bar{x}, y+\bar{y}, t+\bar{t}+2 \sum_{i=1}^{n}\left(x_{i} \bar{y}_{i}-y_{i} \bar{x}_{i}\right)\right)
$$

where $\xi=\left(x_{1}, x_{2}, \ldots, x_{n}, y_{1}, y_{2}, \ldots, y_{n}, t\right):=(x, y, t):=(z, t) \in R^{2 n} \times R, \bar{\xi}=(\bar{z}, \bar{t})$. The group $H^{n}$ plays important roles as $R^{n}$ in conformal geometry, several complex geometry, and harmonic analysis (e.g., see Folland and Stein [2]).

The left-invariant vector fields on $H^{n}$ are of the form

$$
X_{j}=\frac{\partial}{\partial x_{j}}+2 y_{j} \frac{\partial}{\partial t}, \quad Y_{j}=\frac{\partial}{\partial y_{j}}-2 x_{j} \frac{\partial}{\partial t}, \quad j=1,2, \ldots, n, \quad T=\frac{\partial}{\partial t}
$$

The family $\left\{X_{1}, \ldots, X_{n}, Y_{1}, \ldots, Y_{n}\right\}$ satisfies Hörmander's rank condition (see [4]). The Heisenberg gradient of a smooth function $u$ is defined by

$$
\nabla_{H} u=\left(X_{1} u, \ldots, X_{n} u, Y_{1} u, \ldots, Y_{n} u\right)
$$


The divergence of a smooth vector value function $F=\left(F_{1}, \ldots, F_{2 n}\right)$ on $H^{n}$ is defined by

$$
\nabla_{H} \cdot F=X_{1} F_{1}+\cdots+X_{n} F_{n}+Y_{1} F_{n+1}+\cdots+Y_{n} F_{2 n}
$$

For $F=\left(F_{1}, \ldots, F_{2 n+1}\right)$, the usual divergence $\operatorname{div} F$ on $R^{2 n+1}$ is

$$
\operatorname{div} F=\frac{\partial F_{1}}{\partial x_{1}}+\cdots+\frac{\partial F_{n}}{\partial x_{n}}+\frac{\partial F_{n+1}}{\partial y_{1}}+\cdots+\frac{\partial F_{2 n}}{\partial y_{n}}+\frac{\partial F_{2 n+1}}{\partial t}
$$

The norm $|\xi|_{H}$ for $\xi \in H^{n}$ is

$$
|\xi|_{H}=\left(\left(x^{2}+y^{2}\right)^{2}+t^{2}\right)^{\frac{1}{4}}
$$

With this norm, the distance between two points $\xi$ and $\eta$ in $H^{n}$ is defined by

$$
d_{H}(\xi, \eta)=\left|\eta^{-1} \circ \xi\right|_{H}
$$

where $\eta^{-1}$ denotes the inverse of $\eta$ with respect to $\circ$, that is $\eta^{-1}=-\eta$.

The sphere of radius $r>0$ centered at the origin $o=(0,0)$ of $H^{n}$ is the set

$$
S_{H}(o, r)=\left\{\xi \in H^{n}: d_{H}(\xi, o)=r\right\},
$$

and the open ball of radius $r>0$ centered at $o$ is the set

$$
B_{H}(o, r)=\left\{\xi \in H^{n}: d_{H}(\xi, o)<r\right\} .
$$

From [3] we know that the area of $S_{H}(o, r)$ is

$$
\left|S_{H}(o, r)\right|=\wp \alpha_{\wp} r^{2 n+1},
$$

where $\alpha_{\wp}$ is the volume of $B_{H}(o, 1)$.

For simplicity, we denote $B_{H}(o, r)$ and $S_{H}(o, r)$ by $B_{r}$ and $S_{r}$, respectively.

The value of $\alpha_{\wp}$ can be concretely computed. In fact, let

$$
z=\rho z^{\prime} \text { for }\left|z^{\prime}\right|=1 \text {. }
$$

Then

$$
\begin{aligned}
\alpha_{\wp} & =\left|B_{H}(0,1)\right|=\int_{|z|^{4}+t^{2}<1} d z d t=\frac{2 \pi^{n}}{\Gamma(n)} \int_{|\rho|^{4}+t^{2}<1} \rho^{2 n-1} d \rho d t \\
& =\frac{\pi^{n}}{\Gamma(n)} \int_{r^{2}+t^{2}<1, r>0} r^{n-1} d r d t=\frac{2 \pi^{n}}{\Gamma(n)} \int_{0}^{1} \int_{0}^{\frac{\pi}{2}} s^{n} \sin ^{n-1} \theta d s d \theta \\
& =\frac{2 \pi^{n}}{(n+1) \Gamma(n)} \int_{0}^{\frac{\pi}{2}} \sin ^{n-1} \theta d \theta=\frac{\pi^{n}}{(n+1) \Gamma(n)} B\left(\frac{n}{2}, \frac{1}{2}\right) \\
& =\frac{\pi^{n+\frac{1}{2}}}{(n+1) \Gamma(n)} \frac{\Gamma\left(\frac{n}{2}\right)}{\Gamma\left(\frac{n}{2}+1\right)} .
\end{aligned}
$$

Now we first introduce two well-known lemmas. 
Lemma 2.1 (Lagrange's identity [7]) For any two sequences $\left\{a_{i}\right\}$ and $\left\{b_{i}\right\}$, we have

$$
\left(\sum_{i=1}^{n} a_{i}{ }^{2}\right)\left(\sum_{i=1}^{n} b_{i}{ }^{2}\right)=\left(\sum_{i=1}^{n} a_{i} b_{i}\right)^{2}+\sum_{1 \leq i<j \leq n}\left(a_{i} b_{j}-a_{j} b_{i}\right)^{2} .
$$

Lemma 2.2 (The divergence formula in $H^{n}$ [2]) Let $\Omega_{0}$ be a bounded domain in $H^{n}$ with $C^{1}$ boundary $\partial \Omega_{0}$, and let $v$ denote the unit outward normal to $\partial \Omega_{0}$. For any $C^{1}\left(\Omega_{0}\right)$ vector field $V=\left(V_{1}, \ldots, V_{2 n}\right)$, we have

$$
\int_{\Omega_{0}} \nabla_{H} \cdot V d z d t=\int_{\partial \Omega_{0}} M V \cdot v d H
$$

where

$$
M=\left(\begin{array}{cc}
I_{n} & 0 \\
0 & I_{n} \\
2 y & -2 x
\end{array}\right)_{(2 n+1) \times 2 n}
$$

and $I_{n}$ is the identity matrix of $R^{n}$.

Based on these lemmas, we get the following critical estimate.

Lemma 2.3 For any $C^{1}\left(B_{r}\right)$ vector field $V=\left(V_{1}, \ldots, V_{2 n}\right)$, we have

$$
\|M V \cdot v\|_{L^{1}\left(S_{r}\right)}^{2} \leq \wp \alpha_{\wp} r^{2 n+1}\left(4 r^{2}+1\right)\|V\|_{L^{2}\left(S_{r}\right)}^{2} .
$$

Proof A direct calculation shows that

$$
M V=\left(V_{1}, V_{2}, \ldots, V_{2 n}, 2\left(y_{1} V_{1}+\cdots+y_{n} V_{n}\right)-2\left(x_{1} V_{n+1}+\cdots+x_{n} V_{2 n}\right)\right) .
$$

Then by (2.5) we have

$$
\begin{aligned}
|M V|^{2}-|V|^{2} & =4\left(\left(y_{1} V_{1}+\cdots+y_{n} V_{n}\right)-\left(x_{1} V_{n+1}+\cdots+x_{n} V_{2 n}\right)\right)^{2} \\
& \leq 4\left(y_{1}{ }^{2}+\cdots+y_{n}{ }^{2}+\left(-x_{1}\right)^{2}+\cdots+\left(-x_{n}\right)^{2}\right)\left(V_{1}{ }^{2}+\cdots+V_{n}{ }^{2}\right) \\
& \leq 4|\xi|_{H}^{2}|V|^{2},
\end{aligned}
$$

that is,

$$
|M V|^{2} \leq\left(4|\xi|_{H}^{2}+1\right)|V|^{2}
$$

By (2.6) and Hölder's inequality we have

$$
\|M V \cdot v\|_{L^{1}\left(S_{r}\right)}^{2} \leq \wp \alpha_{\wp} r^{2 n+1}\|M V\|_{L^{2}\left(S_{r}\right)}^{2} .
$$

Combining (2.8), we obtain (2.7). 


\section{Picone-type differential inequality and Sturm-type comparison principle}

In this section, we derive a Picone-type differential inequality (Lemma 3.1), and then we prove a Sturm-type comparison principle (Lemma 3.2).

Lemma 3.1 (Picone-type differential inequality) Let $v(z, t)$ and $u(r)$ be the solutions of Eqs. (1.1) and (1.2) respectively, and denote

$$
W(z, t)=\frac{1}{f(v)}\left(A \nabla_{H} v\right)(z, t)+\frac{1}{2 k} B(z, t) .
$$

If there exists $a>0$ such that

$$
v(z, t) \neq 0 \quad \text { for }(z, t) \in G[a,+\infty)
$$

and

$$
p(r) \neq\left(4 r^{2}+1\right) g(r) \quad \text { for } r \in[a,+\infty),
$$

then

$$
\begin{aligned}
& \left(p(r) u^{\prime}(r) u(r)-u^{2}(r) \int_{S_{r}} M W(z, t) \cdot v d H\right)^{\prime} \\
& \geq\left(\frac{u(r)}{\sqrt{\left(4 r^{2}+1\right) g(r)}} \int_{S_{r}} M W(z, t) \cdot v d H-u^{\prime}(r) \sqrt{\left(4 r^{2}+1\right) g(r)}\right)^{2} \\
& \quad+\left(p(r)-\left(4 r^{2}+1\right) g(r)\right)\left(u^{\prime}(r)-\frac{b(r) u(r)}{2\left(p(r)-\left(4 r^{2}+1\right) g(r)\right)}\right)^{2} \\
& \quad+\left(Q(r)-\frac{b^{2}(r)}{4\left(p(r)-\left(4 r^{2}+1\right) g(r)\right)}-c(r)\right) u^{2}(r) .
\end{aligned}
$$

Proof Without loss of generality, we assume that

$$
v(z, t)>0 \text { for }(z, t) \in G[a,+\infty) .
$$

In view of Eq. (1.1), we get

$$
\begin{aligned}
\nabla_{H} & \cdot W(z, t) \\
= & \nabla_{H} \cdot\left(\frac{1}{f(v)}\left(A \nabla_{H} v\right)(z, t)+\frac{1}{2 k} B(z, t)\right) \\
= & \nabla_{H}\left(\frac{1}{f(v)}\right) \cdot A \nabla_{H} v+\frac{1}{f(v)} \nabla_{H} \cdot\left(A \nabla_{H} v\right)+\frac{1}{2 k} \nabla_{H} \cdot B \\
= & \left(\frac{1}{f(v)}\right)^{\prime} \nabla_{H} v \cdot A \nabla_{H} v-\frac{1}{f(v)}\left(B^{T} \nabla_{H} v+C f(v)\right)+\frac{1}{2 k} \nabla_{H} \cdot B \\
= & -\frac{f^{\prime}(v)}{f^{2}(v)} \nabla_{H} v \cdot A \nabla_{H} v-\frac{1}{f(v)} B^{T} \nabla_{H} v-C+\frac{1}{2 k} \nabla_{H} \cdot B \\
\leq & -k\left(W-\frac{1}{2 k} B\right)^{T} A^{-1}\left(W-\frac{1}{2 k} B\right)-B^{T} A^{-1}\left(W-\frac{1}{2 k} B\right)-C+\frac{1}{2 k} \nabla_{H} \cdot B \\
= & -k W^{T} A^{-1} W+\frac{1}{4 k} B^{T} A^{-1} B-C+\frac{1}{2 k} \nabla_{H} \cdot B .
\end{aligned}
$$


Now we calculate the left-hand side of (3.1). By combining (3.2) and (2.6) it follows that

$$
\begin{aligned}
& \left(p(r) u^{\prime}(r) u(r)-u^{2}(r) \int_{S_{r}} M W(z, t) \cdot v d H\right)^{\prime} \\
& =p u^{\prime 2}+\left(p u^{\prime}\right)^{\prime} u-2 u u^{\prime} \int_{S_{r}} M W \cdot v d H-u^{2} \int_{\partial S_{r}} M W \cdot v d s \\
& =p u^{\prime 2}-b u u^{\prime}-c u^{2}-2 u u^{\prime} \int_{S_{r}} M W \cdot v d H-u^{2} \int_{S_{r}} \nabla_{H} \cdot W d H \\
& \geq p u^{\prime 2}-b u u^{\prime}-c u^{2}-2 u u^{\prime} \int_{S_{r}} M W \cdot v d H \\
& \quad-u^{2} \int_{S_{r}}\left(-k W^{T} A^{-1} W+\frac{1}{4 k} B^{T} A^{-1} B-C+\frac{1}{2 k} \nabla_{H} \cdot B\right) d H \\
& =p u^{\prime 2}-b u u^{\prime}-c u^{2}-2 u u^{\prime} \int_{S_{r}} M W \cdot v d H+k u^{2} \int_{S_{r}} W^{T} A^{-1} W d H+u^{2} Q(r) .
\end{aligned}
$$

By $\left(C_{1}\right)$ and (2.7) this implies that

$$
\int_{S_{r}} W^{T} A^{-1} W d H \geq \lambda^{-1} \int_{S_{r}} W^{2} d H \geq \frac{1}{\lambda \wp \alpha_{\wp} r^{2 n+1}\left(4 r^{2}+1\right)}\left(\int_{S_{r}} M W \cdot v d H\right)^{2} .
$$

Recalling $g(r)$ in (1.6), we have

$$
\begin{aligned}
& \left(p(r) u^{\prime}(r) u(r)-u^{2}(r) \int_{S_{r}} M W \cdot v d H\right)^{\prime} \\
& \geq p u^{\prime 2}-b u u^{\prime}-c u^{2}-2 u u^{\prime} \int_{S_{r}} M W \cdot v d H+\frac{u^{2}}{\left(4 r^{2}+1\right) g}\left(\int_{S_{r}} M W \cdot v d H\right)^{2}+u^{2} Q \\
& =\left(p-\left(4 r^{2}+1\right) g\right)\left(u^{\prime}-\frac{b u}{2\left(p-\left(4 r^{2}+1\right) g\right)}\right)^{2}+\left(Q-\frac{b^{2}}{4\left(p-\left(4 r^{2}+1\right) g\right)}-c\right) u^{2} \\
& \quad+\left(\frac{u}{\sqrt{\left(4 r^{2}+1\right) g}} \int_{S_{r}} M W \cdot v d H-u^{\prime} \sqrt{\left(4 r^{2}+1\right) g}\right)^{2} .
\end{aligned}
$$

Lemma 3.2 (Sturm-type comparison principle) Let $u(r)$ be a nontrivial solution of Eq. (1.2), and let $a_{1}$ and $a_{2}$ be two adjacent zeros of $u(r)$. If

$$
p(r) \neq\left(4 r^{2}+1\right) g(r)
$$

and

$$
\begin{aligned}
& \int_{a_{1}}^{a_{2}}\left(Q(r)-\frac{b^{2}(r)}{4\left(p(r)-\left(4 r^{2}+1\right) g(r)\right)}-c(r)\right) u^{2}(r) d r \\
& \quad+\int_{a_{1}}^{a_{2}}\left(p(r)-\left(4 r^{2}+1\right) g(r)\right)\left(u^{\prime}(r)-\frac{b(r) u(r)}{2\left(p(r)-\left(4 r^{2}+1\right) g(r)\right)}\right)^{2} d r>0
\end{aligned}
$$

then any solution $v(z, t)$ of Eq. (1.1) has at least one zero on $G\left[a_{1}, a_{2}\right]$. 
Proof We use the contradiction and suppose that there exists a nontrivial solution $v(z, t)$ of Eq. (1.1) such that

$$
v(z, t)>0 \quad \text { on } G\left[a_{1}, a_{2}\right]
$$

Integrating (3.1) in Lemma 3.1 from $a_{1}$ to $a_{2}$ gives

$$
\begin{aligned}
& \int_{a_{1}}^{a_{2}}\left(p u^{\prime} u-u^{2} \int_{S_{r}} M W \cdot v d H\right)^{\prime} d r \\
& \geq \int_{a_{1}}^{a_{2}}\left(\frac{u}{\sqrt{\left(4 r^{2}+1\right) g}} \int_{S_{r}} M W \cdot v d H-u^{\prime} \sqrt{\left(4 r^{2}+1\right) g}\right)^{2} \\
& \quad+\int_{a_{1}}^{a_{2}}\left(Q-\frac{b^{2}}{4\left(p-\left(4 r^{2}+1\right) g\right)}-c\right) u^{2} \\
& \quad+\left(p-\left(4 r^{2}+1\right) g\right)\left(u^{\prime}-\frac{b u}{2\left(p-\left(4 r^{2}+1\right) g\right)}\right)^{2} .
\end{aligned}
$$

Noting that the left-hand side is 0 , this yields

$$
\int_{a_{1}}^{a_{2}}\left(Q-\frac{b^{2}}{4\left(p-\left(4 r^{2}+1\right) g\right)}-c\right) u^{2}+\left(p-\left(4 r^{2}+1\right) g\right)\left(u^{\prime}-\frac{b u}{2\left(p-\left(4 r^{2}+1\right) g\right)}\right)^{2} \leq 0,
$$

which contradicts to (3.4). The proof is completed.

An immediate consequence of Lemma 3.2 is the following:

Corollary 3.3 Let $u(r)$ be a nontrivial solution of Eq. (1.2), and let $a_{1}$ and $a_{2}$ be two adjacent zeros of $u(r)$. If there exists $a>0$ such that for any $r \in[a,+\infty)$,

$$
\begin{aligned}
& p(r)>\left(4 r^{2}+1\right) g(r), \\
& Q(r) \geq \frac{b^{2}(r)}{4\left(p(r)-\left(4 r^{2}+1\right) g(r)\right)}+c(r),
\end{aligned}
$$

and the equality is not always true in any subinterval of $[a,+\infty)$, then any solution $v(z, t)$ of Eq. (1.1) has at least one zero on $G\left[a_{1}, a_{2}\right]$.

\section{Proof of Theorem 1.1}

Proof of Theorem 1.1 Let $u(r)$ be an oscillatory solution of Eq. (1.2), and let $r_{i}$ be the sequence of zeros of $u(r)$ satisfying

$$
r_{0} \leq r_{1}<r_{2}<\cdots<r_{i} \cdots, \quad \lim _{i \rightarrow \infty} r_{i}=\infty
$$

For every $G_{i}=\left\{(z, t) \in H^{n}: r_{i}<|(z, t)|_{H}<r_{i+1}\right\}$, by Corollary 3.3 it follows that every solution $v(z, t)$ of Eq. (1.1) has at least one zero on $\bar{G}_{i}$, which shows that $v(z, t)$ is oscillatory. From the arbitrariness of $v(z, t)$ we claim that Eq. (1.1) is oscillatory. 
Acknowledgements

The authors are greatly indebted to Xinjing Wang and Manli Song for the insightful comments and wise advices.

\section{Funding}

This work was completed with the support of the National Natural Science Foundation of China (No. 11771354).

\section{Availability of data and materials}

Not applicable.

\section{Competing interests}

The authors declare that they have no competing interests.

\section{Authors' contributions}

All authors contributed equally. All authors read and approved the final manuscript.

\section{Publisher's Note}

Springer Nature remains neutral with regard to jurisdictional claims in published maps and institutional affiliations.

Received: 16 January 2021 Accepted: 28 March 2021 Published online: 07 April 2021

\section{References}

1. El-Sayed, M.: An oscillation criteria for a forced second-order linear differential equation. Proc. Am. Math. Soc. 24, 169-182 (1982)

2. Folland, G., Stein, E.: Estimate for the $\bar{\partial}_{b}$ complex and analysis on the Heisenberg group. Commun. Pure Appl. Math. 27, 429-522 (1974)

3. Garofalo, N., Lanconelli, E.: Frequency functions on the Heisenberg group, the uncertainty principle and unique continuation. Ann. Inst. Fourier (Grenoble) 40, 313-356 (1990)

4. Hörmander, L.: Hypoelliptic second order differential equations. Acta Math. 119, 141-171 (1967)

5. Jaroš, J., Kusano, T.: A Picone type identity for second order half-linear differential equations. Acta Math. Univ. Comen. 68, 137-151 (1999)

6. Kamenev, I.: Oscillation of solution of a second order differential equations with an "integrally small" coefficient. Differ. Uravn. 13, 2141-2148 (1977)

7. Kuang, J:: Applied Inequalities, 3rd edn. Shangdong Science and Technology Press (2004)

8. Kusano, T., Jaroš, J., Yoshida, N.: A Picone-type identity and Sturmian comparison and oscillation theorems for a class of half-linear partial differential equations of second order. Nonlinear Anal. 40, 381-395 (2000)

9. Misir, A., Tiryaki, A.: Comparison results for nonlinear equations involving a-harmonic operator. Mediterr. J. Math. 13, 4949-4959 (2016)

10. Noussair, E., Swanson, C.: Oscillation of semilinear elliptic inequalities by Riccati transformation. Can. J. Math. 32, 908-923 (1980)

11. Picone, M.: Sui valori eccezionali di un parametron da cui dipend un'equazione differenziale linear ordinaria del second'ordine. Ann. Sc. Norm. Super. Pisa, Cl. Sci. 11, 1-141 (1909)

12. Sturm, C.: Mémoire sur les équations différentielles linéaires du second ordre. J. Math. Pures Appl. 1, 106-186 (1836)

13. Usami, H.: Some oscillation theorems for a class of quasilinear elliptic equations. Ann. Mat. Pura Appl. 175, 277-283 (1998)

14. Xu, Z., Jia, B., Ma, D.: Oscillation theorems for elliptic equations with damping. Appl. Math. Comput. 156, 93-106 (2004)

15. Zhuang, R., Jia, B., Wang, Q.: Sturm comparison theorem of solutions for second order nonlinear elliptic differential equation. Acta Math. Sinica (Chin. Ser.) 60, 1037-1046 (2017)

16. Zhuang, R., Li, W.: Interval oscillation criteria for second order neutral nonlinear differential equations. Appl. Math. Comput. 157, 39-51 (2004)

\section{Submit your manuscript to a SpringerOpen ${ }^{\circ}$ journal and benefit from:}

- Convenient online submission

- Rigorous peer review

- Open access: articles freely available online

- High visibility within the field

- Retaining the copyright to your article

Submit your next manuscript at $\gg$ springeropen.com 